\title{
Zwitterionic AlEgens: Rational Molecular Design for NIR-II Fluorescence Imaging-Guided Synergistic Phototherapy
}

\author{
Wei Zhu, ${ }^{[a, b, c,+]}$ Miaomiao Kang, ${ }^{[a, b, c,+]}$ Qian Wu, ${ }^{[a, b, c]}$ Zhijun Zhang, ${ }^{[a, b, c]}$ Yi Wu, ${ }^{[a]}$ Chunbin Li, ${ }^{[d]}$ Kai Li, ${ }^{*,[e]}$ \\ Lei Wang, ${ }^{[a, b]}$ Dong Wang, ${ }^{*},[a]$ and Ben Zhong Tang ${ }^{*},[c]$
}

[a] Dr. W. Zhu, Dr. M. Kang, Dr. Q. Wu, Dr. Z Zhang, Y. Wu, Professor D. Wang

Center for AIE Research, Shenzhen Key Laboratory of Polymer Science and Technology, Guangdong Research Center for Interfacial Engineering of

Functional Materials, College of Materials Science and Engineering, Shenzhen University, Shenzhen, 518060, China

E-mail: wangd@szu.edu.cn.

[b] Dr. W. Zhu, Dr. M. Kang, Dr. Q. Wu, Dr. Z. Zhang

Key Laboratory of Optoelectronic Devices and Systems of Ministry of Education and Guangdong Province, College of Physics and Optoelectronic

Engineering, Shenzhen University, Shenzhen, 518060, China

[c] Dr. W. Zhu, Dr. M. Kang, Dr. Q. Wu, Dr. Z. Zhang, Professor B. Z. Tang

Department of Chemistry, Hong Kong Branch of Chinese National Engineering Research Center for Tissue Restoration and Reconstruction, The Hong Kong University of Science and Technology, Clear Water Bay, Kowloon, 999077, Hong Kong, China

E-mail: tangbenz@ust.hk.

[d] C. Li

Institute of Biomedicine and Biotechnology, Shenzhen Institutes of Advanced Technology, Chinese Academy of Sciences, Shenzhen 518055, China

[e] Professor K. Li

Department of Biomedical Engineering, Southern University of Science and Technology, Shenzhen 518055, China.

[+] These authors contributed equally to this work.

Supporting information for this article is given via a link at the end of the document.

\begin{abstract}
Fluorescence imaging in the second near-infrared region (NIR-II) can penetrate tissue at centimeter depths and obtain high fidelity of images. However, facile synthesis of small-molecule fluorescent photosensitizers for efficient NIR-II fluorescence imaging as well as photodynamic and photothermal combinatorial therapies (PDT-PTT) is still a challenging task. Herein, we reported a rational design and facile synthesis protocol for a series of novel NIR-emissive zwitterionic luminogens with aggregation-induced emission (AIE) features for cancer phototheranostics. Consistent with the intrinsic features including long emission wavelength, effective reactive oxygen species (ROS) generation, and excellent photothermal conversion efficiency (35.76\%), in vitro and in vivo evaluation showed one of these presented AIE luminogens provided excellent performance in NIR-II fluorescence imaging-guided synergistic phototherapy against cancer.
\end{abstract}

Fluorescence imaging (FLI) has captivated much interest for both fundamental study and practical application in the field of biomedicine, by virtue of its intrinsic advantages including noninvasive detection, superb sensitivity, in situ workability and high temporal resolution. ${ }^{[1]}$ Of particular interest in this area is FLI in the second near-infrared wavelength range (NIR-II, 1000-1700 nm), which allows deepened penetration, diminished tissue autofluorescence, and minimized photo-damage to the biological structure. ${ }^{\left[{ }^{2]}\right.}$ Evidently, NIR-II FLI represents a vitally powerful protocol for high-clarity and deep visualization of tissues/organs ranging from 2D to $3 \mathrm{D}$, comparing with FLI in well-studied visible and NIR-I $(700-900 \mathrm{~nm})$ window. ${ }^{[3]}$ Although some NIR-II fluorophores have been explored, the current situation is still far from ideal. Notwithstanding the high brightness of inorganic fluorescent nanomaterials, they are usually suffering from toxicity concerns, which severely restrict their biological applications. ${ }^{[4]}$ As alternatives, organic fluorophores constructed by biologically inert components are potentially promising in NIR-II FLI, however, the emission is easily quenched as aggregates due to the dominated nonradiative decay caused by intense intermolecular $\pi-\pi$ interactions ${ }^{[5]}$ Given the circumstances, as a burgeoning concept, aggregation-induced emission (AIE) provides a new design viewpoint and direction for the development of NIR-II FLI agents. AIE refers to a unique photophysical phenomenon that a family of fluorophores shows faintly emissive or quenched emission in the molecularly dissolved state but they emit intensively in aggregates. ${ }^{[6]}$ The AIE tendency endows AIE-active luminogens (AIEgens) with several attractive features for FLI application, such as high brightness as nanoaggregates, high photobleaching threshold, and large Stokes shift. ${ }^{[7]}$ The combination of AIE concept and NIR-II FLI definitely demonstrates a win-win co-operation towards bioapplications.

Enthusiastic efforts have been recently devoted to exploring AIE-active luminogens (AIEgens) for NIR-II FLI, although a handful of capable AIEgens were developed, these reported AIEgens exhibit their respective and collective drawbacks. ${ }^{[8]}$ Common synthetic protocols of these AIEgens generally require tedious several-step reactions and inconvenient purifications, which supremely impede their practical applications. ${ }^{[8 \mathrm{a}, 8 \mathrm{~b}]}$ Moreover, most NIR-II AIEgens adopt an electron donating-accepting-donating (D-A-D) type structure with benzo[1,2-c:4,5-c']bis([1,2,5]thiadiazole) (BBTD) as electron accepting unit, therefore, further improvement on molecular diversity is still called for. ${ }^{[8 c, 8 d]}$ Additionally, in view of the fact that FLI-guided phototherapy involving photodynamic therapy (PDT) and/or photothermal therapy (PTT) has been recognized to be a promising strategy for simultaneous disease diagnosis and therapy, especially for cancer theranostics, ${ }^{[9]}$ developing effective AIE photosensitizer for NIR-II FLI-guided synergistic phototherapy remains an urgently needed task with very limited success.

In this contribution, we demonstrated for the first time the rational design and facile synthesis of zwitterion-type NIR AIEgens with good compatibility, high reactive oxygen species (ROS) generation, and extraordinary photothermal conversion efficiency. Experimental outcomes revealed that one of these novel AIEgens performed well in NIR-II FLI-guided phototheranostics for cancer treatment. This study provides a molecular guideline to develop multifunctional theranostic agent. 


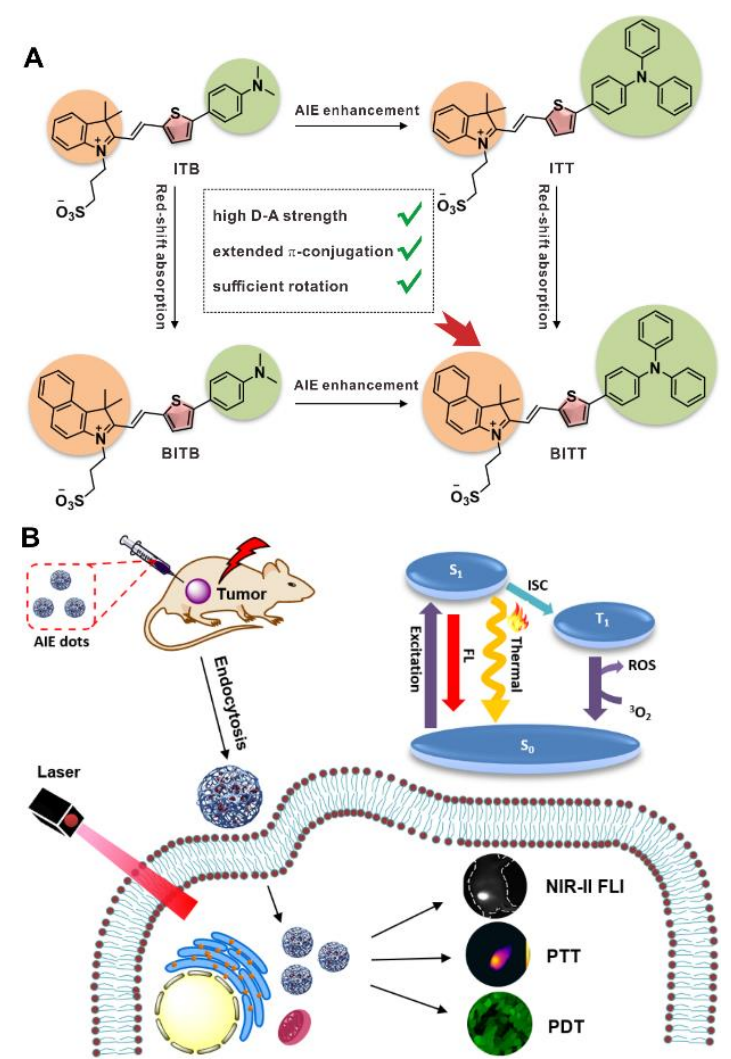

Figure 1. (A) Rational design strategy of AIEgens (ITB, ITT, BITB, and BITT). (B) Schematic illustration of using BITT dots for NIR-II FLI-guided synergistic phototherapy against cancer.

As illustrated in Figure 1A and Scheme S1, zwitterionic compounds BITT, BITB, ITT, and ITB comprising of dimethylaniline/ triphenylamine moiety (working as $D$ ), thiophene fragment ( $D$ and $\pi$-bridge), double bond ( $\pi$-bridge) and the quaternary ammonium salt unit (A) were facilely synthesized through a few-step-reactions. Suzuki-Miyaura coupling reaction between 4-bromo- $N, N$-diphenylaniline/4-bromo- $N, N$-dimethylaniline and (5-form-ylthiophen-2-yl)boronic acid smoothly proceeded, producing intermediate aldehydes 1 and 3 in yield of $74 \%$ and $48 \%$, respectively. Knoevenagel condensation reaction of aldehydes with pre-synthesized $\mathbf{2}$ or $\mathbf{4}$ was further conducted and target compounds BITT, BITB, ITT, and ITB were obtained in moderate yields.

The single crystals of BITT and ITT that were obtained by slow evaporation of their solvent mixture of DCM and hexane (2:1, v/v) at ambient temperature were tested for deciphering of the optical properties (Figure S1 and Tables S1). As depicted in Figure S2, the intermolecular distances between two parallel planes were as large as $4.841 \AA$ and $4.135 \AA$ (for BITT) and $4.194 \AA$ and $3.648 \AA$ (for ITT), respectively, which essentially prevented emission quenching in its aggregate state caused by intermolecular $\pi-\pi$ interaction. It was believed that the large intermolecular distances could be attributed to the existences of both twisted triphenylamine moiety and alkyl chain with sulfonic acid group. Moreover, multiple inter- and intramolecular interactions (such as $\mathrm{C}-\mathrm{H} \cdots \mathrm{O}, \mathrm{C}-\mathrm{H} \cdots \mathrm{S}$, and $\mathrm{C}-\mathrm{H} \cdots \mathrm{m}$ ) in the crystal lattices were capable of rigidifying the molecular conformation and restraining molecular motions. Furthermore, BITT and ITT structurally possessed several molecular rotators, which can freely rotate in solution state, and thus consume the excited-state energy upon photoexcitation, consequentially making them non-emissive or weekly emissive in solution. It seems reasonable to infer that BITT and ITT are potentially AIE-active.

The optical properties of these compounds were characterized by UV-Vis and photoluminescence (PL) spectroscopies (Table S2). BITT and ITT possessed roughly the same maximum absorption peaks at around $590 \mathrm{~nm}$. Besides, the absorptions of BITB and ITB were almost the same peaking at around $630 \mathrm{~nm}$ (Figure 2A). We further used a mixed solvent (ethanol/hexane) with different hexane fractions as a solvent system to evaluate the AIE features of BITT, BITB, ITT, and ITB (Figure S3). As depicted in Figure 2B, along with continuously raising the fraction of hexane, the PL intensities of these four compounds dramatically increased upon the formation of aggregates. The maxima of emission intensity, achieved at $95 \%$ of hexane fraction, were about 11.3, 2.3, 12.9, and 4.0 times higher than those in pure ethanol solution, respectively. These outcomes strongly demonstrated that all these four synthesized compounds were typical AIEgens. The AIE tendencies of BITT and ITT were much better than both BITB and ITB, mainly because the existence of triphenylamine unit endowed BITT and ITT with both more molecular rotors and twisted formation, which could result in weaker emission in solution, as well as boosted emission intensity in aggregates. In addition, the maximum emissions of BITT, BITB, ITT, and ITB in solution were located at $731,752,722$, and $741 \mathrm{~nm}$, respectively, with quantum yields of $5.8 \%, 6.2 \%, 4.4 \%$, and $4.7 \%$. Notably, the PL spectra of BITT and ITT in the solid state were respectively peaked at 818 and $829 \mathrm{~nm}$ with part of the tail locating in the NIR-II region (Figure $2 \mathrm{C}$ ), and their fluorescence decay curves reveal that their lifetimes are 0.88 and 0.81 ns (Figure S4). To better understand the optical properties of these AIEgens, density functional theory (DFT) calculations were carried out (Figure S5). DFT calculations revealed that the long absorption wavelength is ascribed to its small HOMO-LOMO energy gap due to the stronger D-A interaction and extended $\pi$-conjugation. Besides, these AIEgens also exhibit typical positive solvatochromism due to the typical D-A 
structure. The solvatochromism study displayed that with the increase of solvent polarity, the emission maximum of AlEgens largely redshifted, while the emission intensity was considerably decreased (Figure S6), suggesting a strong twisted intramolecular charge transfer (TICT) effect.

It has been demonstrated that strong D-A effect could lead to the separation of HOMO-LUMO distribution and reduced singlet-triplet energy gap, which significantly favors the ROS generation. ${ }^{[10]}$ Encouraged by that, the abilities of ROS generation of these AIEgens were evaluated by using dichlorofluorescein (DCFH-DA) as a probe. It was observed that DCFH-DA alone was nearly non-emissive, while the fluorescence intensity rapidly increased along with the increase of the irradiation time by using laser irradiation ( $660 \mathrm{~nm}, 0.3$ $\mathrm{W} / \mathrm{cm}^{2}$ ) in the presence of AIEgens (Figure S7). Figure 2D showed that BITT was superior to the others in terms of ROS production, in this case, the emission intensity of DCFH-DA was remarkably boosted with 615 -fold enhancement. Additionally, the photothermal conversion performance of these presented AIEgens was also estimated. The influence of different laser power density $(0.2,0.3$, and $\left.0.4 \mathrm{~W} / \mathrm{cm}^{2}\right)$ on the temperature changes of BITT in DMSO $\left(3 \times 10^{-4} \mathrm{M}\right)$ was first investigated (Figure S8a). It was found that the temperature of BITT solutions elevated quickly with increasing the power density of the laser. When the laser power density increased to $0.3 \mathrm{~W} / \mathrm{cm}^{2}$, the temperature of the solution rose rapidly to around $85{ }^{\circ} \mathrm{C}$ under $660 \mathrm{~nm}$ laser irradiation for 5 min. Further, the temperature of BITT was effectively and quickly elevated in a concentration-dependent manner under $660 \mathrm{~nm}$ laser irradiation at 0.3 $\mathrm{W} / \mathrm{cm}^{2}$ (Figure S8b). From the above, the photothermal conversion efficiency of AlEgens was investigated under a reasonable concentration $\left(3 \times 10^{-4} \mathrm{M}\right)$ and laser power density $\left(0.3 \mathrm{~W} / \mathrm{cm}^{2}\right)$. As depicted in Figure 2E and S9, BITT showed the best performance in the photothermal effect among these AIEgens. The superior photothermal efficiency of BITT could be attributed to its more sufficient rotators than other AlEgens.
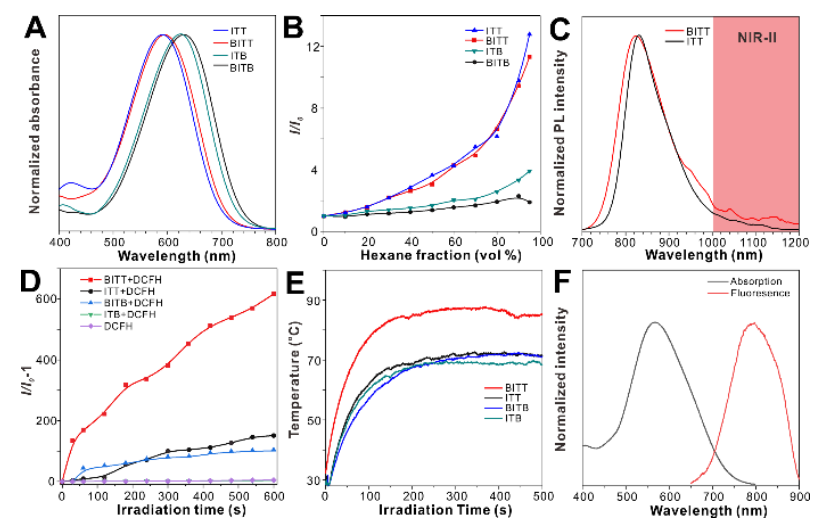

Figure 2. (A) Normalized absorption spectra of AlEgens dissolved in ethanol. (B) Plots of relative PL intensity ( $/ / 10)$ of $A$ IEgens versus hexane fraction. (C) Normalized photoluminescence spectra of BITT and ITT as solid. (D) ROS generation of AIEgens $\left(1 \times 10^{-6} \mathrm{M}\right)$ upon laser irradiation $\left(660 \mathrm{~nm}, 0.3 \mathrm{~W} / \mathrm{cm}^{2}\right)$ using DCFH-DA as an indicator. (E) Photothermal conversion behavior of AIEgens at $3 \times 10^{-4} \mathrm{M}$ under $660 \mathrm{~nm}$ irradiation at $0.3 \mathrm{~W} / \mathrm{cm}^{2}$. (F) The absorption and emission spectra of BITT dots.

Aiming to effectively conduct bioapplications, water-soluble BITT dots suspension was fabricated through nanoprecipitation. The particle size of nanoparticles in aqueous solution was confirmed by dynamic light scattering (DLS) analysis (size $=69.86 \mathrm{~nm}$ ). Transmission electron microscope (TEM) analysis revealed that BITT dots have uniform spherical morphology (Figure S10). The aqueous dispersion of BITT dots displayed a maximum absorption band at $575 \mathrm{~nm}$ and a maximum emission band at $797 \mathrm{~nm}$ (Figure $2 \mathrm{~F}$ ). BITT dots exhibit excellent performance in both ROS generation and photothermal effect (Figure S11). Furthermore, the photothermal stability of the photothermal agent was investigated. In this study, BITT dots in aqueous solution $\left(1.8 \times 10^{-4} \mathrm{M}\right)$ suffered from 9 successive warming-cooling cycles. The cycle results demonstrated that the highest temperatures were around $55^{\circ} \mathrm{C}$ for all 9 cycles under $660 \mathrm{~nm}$ irradiation at $0.3 \mathrm{~W} / \mathrm{cm}^{2}$ for $5 \mathrm{~min}$. Interestingly, even after 9 cycles, the maximized elevated temperature of the nanoparticles showed negligible changes (Figure S12). The photothermal conversion efficiency $(\eta)$ of BITT dots was determined to be $35.76 \%$, which was much higher than the vast majority of typical photothermal agents (ICG $\approx 3.1 \%$, cyanine dyes $\approx 26.6 \%$, and gold nanorods $\approx 21.0 \%$ ) (Figure S13). ${ }^{[1]}$ All the above-mentioned studies on photothermal and photodynamic properties of BITT dots suggested that the photostable BITT dots represented an excellent candidate serving as a theranostic probe for the NIR-II FLI-guided synergistic PTT-PDT therapy of tumors.

The cellular uptake of the photosensitizer is of special importance for determining the theranostic effect. The cellular uptake experiment of AIEgens in 4T1 cells was investigated as a preliminary study. As depicted in Figure 3A and S14, all of BITT, BITB, ITT, and ITB can be largely uptaken by cells that glowed with a red luminescence under laser radiation. Furthermore, the intracellular localization investigation using nucleus-specific blue fluorescent dye displayed that BITT was mainly localized in the cytoplasm with the optimal performance among these AIEgens. To quantitatively estimate the phototherapeutic effect of BITT on 4T1 cancer cells, a standard CCK8 assay was employed. As depicted in Figure 3B, BITT exhibited negligible dark toxicity, solidly suggesting its good biocompatibility. Upon $660 \mathrm{~nm}$ laser irradiation, the cell viability gradually dropped with increasing BITT concentration, even as low as $8 \%$, revealing high efficiency for cancer cell killing. Additionally, the intracellular ROS generation of BITT was evaluated by utilizing DCFH-DA as an indicator. As illustrated in Figure 3C, 4T1 cells incubated with BITT and DCFH-DA exhibited bright green fluorescence upon $660 \mathrm{~nm}$ laser irradiation, but no obvious fluorescence was observed in the controlled experiments (PBS, PBS+laser and BITT alone). Also, live/dead staining experiments assessed by using fluorescein diacetates (FDA) (green color for live cells) /propidium iodide (PI) (red color for dead cells) revealed that the combination of BITT and laser irradiation powerfully caused both cancer cell apoptosis and necrosis. ${ }^{[12]}$ As shown in Figure 3D, strong green fluorescent signal was observed in the light alone and BITT alone 
groups, indicating the desirable biocompatibility of BITT and the negligible side effect of NIR laser irradiation. By contrast, bright red fluorescent signal was detected in the cells treated with BITT upon NIR laser irradiation, implying that BITT has excellent controllable photoablation ability against cancer cells. These results definitely demonstrated the high phototoxicity of BITT against 4T1 cells, which can be attributed to the efficient ROS generation and excellent photothermal conversion.

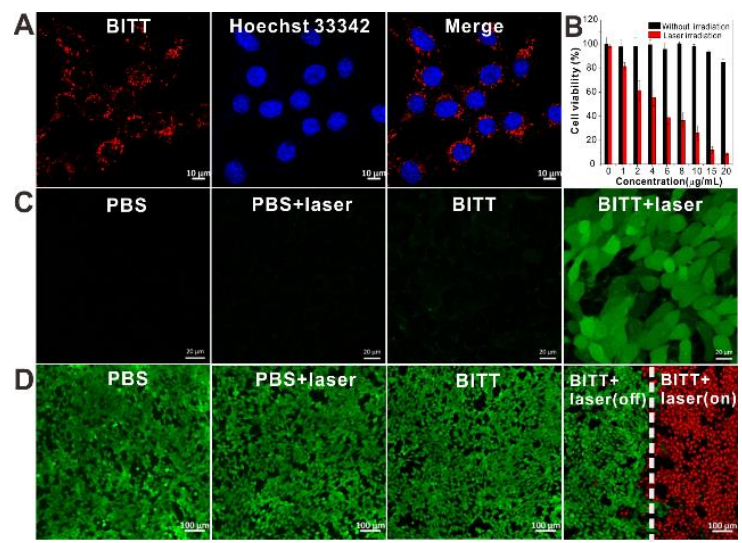

Figure 3. (A) CLSM imaging of $4 \mathrm{~T} 1$ cells after incubation with BITT $\left(1 \times 10^{-5} \mathrm{M}\right)$ for $1 \mathrm{~h}$, red fluorescence corresponds to BITT, nucleus colocalized with the blue fluorescence. Scale bar: $10 \mu \mathrm{m}$. (B) Cell viability of $4 \mathrm{~T} 1$ cells stained with different concentrations of BITT in the absence or presence of laser irradiation. (C) CLSM imaging of intracellular ROS in 4T1 cells using DCFH-DA upon diverse treatments. Scale bar: $20 \mu \mathrm{m}$. (D) Live/dead cell staining of 4T1 cells treated with diverse treatments assessed by FDA (green fluorescence) /PI (red fluorescence). Scale bar: $100 \mu \mathrm{m}$.

In vivo NIR-I and NIR-II FL images were subsequently captured at predetermined time intervals $(0.5,6,12$, and $24 \mathrm{~h})$ post-injection. As is depicted in Figure 4A and 4B, the NIR-I and NIR-II FLI both allowed clear visualization of the tumors in mice. Reasonably, with the metabolism of agents, their imaging signals were weakened after $24 \mathrm{~h}$. However, compared with the disappearance of NIR-I FLI signal, the NIR-II FLI of the tumors on the mice was determined to be brighter and more legible because of immensely reducing the autofluorescence of tissues, indicating that the NIR-II FLI with superior imaging resolution and high signal/background ratio (SBR) can provide more realistic and reliable imaging results. In the preliminary in vivo phototherapeutic experiment, the tumor surface temperature was studied by intratumor injection of BITT dots into 4T1 tumor-bearing nude mice. After injection, these mice were exposed to $660 \mathrm{~nm}$ laser irradiation at $0.3 \mathrm{~W} / \mathrm{cm}^{2}$ for $10 \mathrm{~min}$. The tumor surface temperature of BITT dots group rapidly rose to $52.4{ }^{\circ} \mathrm{C}$ after $2 \mathrm{~min}$ of irradiation which was sufficient to destroy the tumor cells effectively (Figure S15). In contrast, the temperature on the tumor site of the control group (saline) only slightly increased after laser irradiation, indicating that $660 \mathrm{~nm}$ laser-induced photothermal effect was negligible (Figure S16). ${ }^{[13]}$ To evaluate the actual therapeutic effect of BITT dots in vivo, $4 \mathrm{~T} 1$ tumor-bearing nude mice were randomly divided into four groups (6 mice in per group) for separate treatments as follows: (1) saline, (2) saline+laser, (3) BITT dots, and (4) BITT dots+laser. After $6 \mathrm{~h}$ intratumoral injection, the tumors of mice in each group were continuously irradiated with $660 \mathrm{~nm}$ laser $\left(0.3 \mathrm{~W} / \mathrm{cm}^{2}\right)$ for $10 \mathrm{~min}$. The tumor sizes were carefully monitored during 15 days after irradiation to qualitatively assess the therapeutic effect of each group. The size of tumors in the three control groups clearly exhibited a sustained increase, demonstrating that dots or laser irradiation alone could not suppress the tumor growth at all (Figure S17). Exhilaratingly, as displayed in Figure 4D, the tumors in "BITT dots+laser" group were completely eradicated without regrowth or recurrence during the observation period. Besides, no significant body weight loss was found for all the experimental groups (Figure 4E), revealing very low side effect of BITT dots in vivo. Furthermore, the biosafety of the BITT dots in vivo was evaluated. Blood routine examination and hepatic/renal function analysis of the mice in different treatment groups were systematically conducted, and the obtained results revealed that no hematological toxicity, hepatotoxicity, and nephrotoxicity were observed (Table S3 and Figure S18). The tumors and major organs of mice including heart, liver, spleen, lung, and kidney were harvested and analyzed by hematoxylin and eosin (H\&E) staining after therapy. No noticeable tissue damage or adverse effects were found in these tested organs, verifying the safety of dual phototherapy for living organisms (Figure S19). In addition, the CD31 staining was carried out to study the phototherapy treatment effect on vascular damage. Comparing to the other three control groups, a significant decrease in the intensity of CD31-stained blood vessels (green fluorescence) in "BITT dots+laser" treatment was clearly observed, indicating the alterations in angiogenesis caused by the dual phototherapy. Moreover, to estimate whether cell apoptosis was involved in the therapeutic process on 4T1 tumors, the TUNEL assay was used to quantify cell apoptosis in tumor sections from all four groups. ${ }^{[14]}$ In contrast with three control groups, the "BITT dots+laser" treatment group showed significant cell apoptosis throughout the tumor, confirming the effectiveness of synergistic phototherapy of BITT dots (Figure S20). 


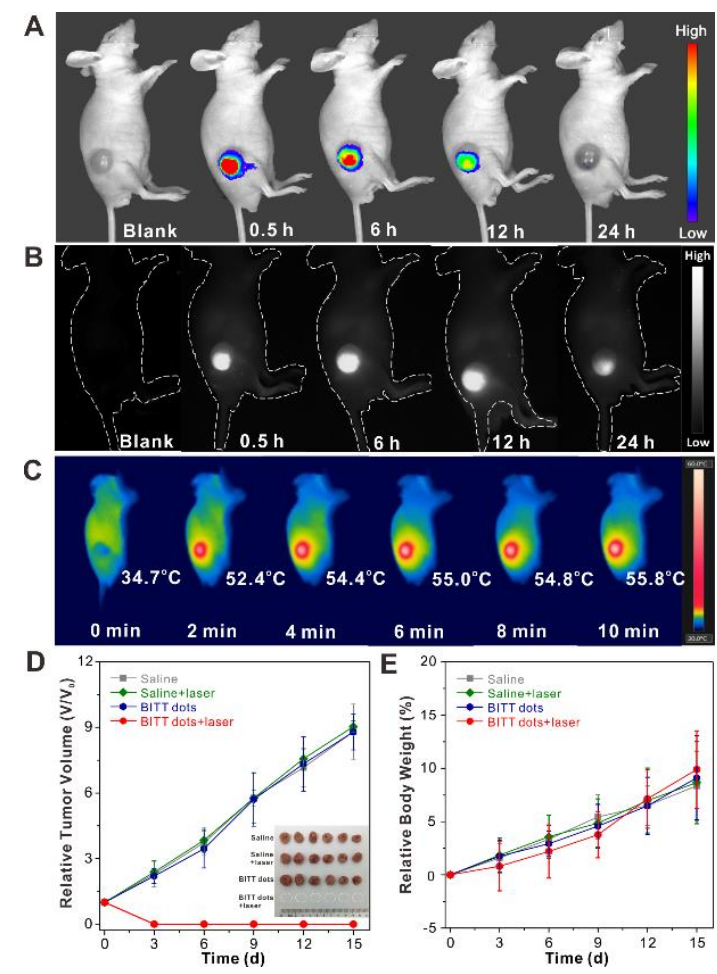

Figure 4. (A) NIR-I FL images and (B) NIR-II FL images of $4 T 1$ tumor-bearing mice at different time points after injection of BITT dots. (C) IR thermal images of $4 T 1$ bearing-tumor mice injected with BITT dots followed by laser irradiation $\left(660 \mathrm{~nm}, 0.3 \mathrm{~W} / \mathrm{cm}^{2}\right)$ for $2 \mathrm{~min}, 4 \mathrm{~min}, 6 \mathrm{~min}, 8 \mathrm{~min}$, and $10 \mathrm{~min}$. (D) Tumor growth curves of mice treated with Saline, Saline+laser, BITT dots, and BITT dots+laser. Insert: tumor images of 4T1 tumor-bearing mice after 15-day different treatments. (E) Bodyweight changes of mice in different treatment groups.

In summary, a series of novel AIE molecules (BITT, BITB, ITT, and ITB) were rationally designed and facilely synthesized. Among all these AIEgens, BITT with rotor twisted structure, high D-A strength, and strong NIR emission, exhibit the most superior ROS generation performance and photothermal conversion efficiency (35.76\%). In vitro experiments demonstrated that BITT possessed negligible dark toxicity and high phototoxicity towards cancer cells. Furthermore, in vivo evaluation demonstrated that the tumor surface temperature rose to $55^{\circ} \mathrm{C}$ under $0.3 \mathrm{~W} / \mathrm{cm}^{2}$ of laser irradiation, while the NIR-I and NIR-II FLI of the mice were well conducted. The tumor elimination of $4 \mathrm{~T} 1$ bearing-tumor mice revealed that BITT dots with excellent biocompatibility displayed an extraordinary anticancer effect through synergistic PDT-PTT phototherapies. These results suggested that the AIEgen BITT has great potential to serve as a single component theranostic probe for the NIR-II FLI-involved theranostics. This study provides a promising strategy for the design of NIR AIEgens and paves a new pathway for efficient cancer treatments.

\section{Acknowledgements}

This work was supported by the Natural Science Foundation for Distinguished Young Scholars of Guangdong Province (2020B1515020011), the Science and Technology Foundation of Shenzhen City (JCYJ20190808153415062), and the Natural Science Foundation of China (21801169). The authors also acknowledge the Instrumental Analysis Center of Shenzhen University.

Keywords: aggregation-induced emission $•$ NIR-II fluorescence imaging $\bullet$ photodynamic therapy $\bullet$ photothermal therapy $\bullet$ anticancer

[1] a) J. M. Idée, S. Louguet, S. Ballet, C. Corot, Quant. Imaging Med. Surg. 2013, 3, 292; b) K. Rajesh, S. S. Weon, S. Kyoung, W. Y. Kim, S. Koo, S. Bhuniya, J. S. Kim, Chem. Soc. Rev. 2015, 44, 6670-6683.

[2] a) S. Zhu, R. Tian, A. L. Antaris, X. Chen, H. Dai, Adv. Mater. 2019, 31, 1900321; b) N. Alifu, A. Zebibula, J. Qi, H. Zhang, C. Sun, X. Yu, D. Xue, J. W. Y. Lam, G. Li, J. Qian, B. Z. Tang, ACS Nano 2018, 12, 11282-11293; c) G. Hong, A. L. Antaris, H. Dai, Nat. Biomed. Eng. 2017, 1, 0010

[3] a) H. Wan, H. Du, F. Wang, H. Dai, Adv. Funct. Mater. 2019, 29, 1900566; b) Y. Duan, B. Liu, Adv. Mater. 2018, 30, 1802394; c) S. Zhu, S. Herraiz, J. Yue, M. Zhang, H. Wan, Q. Yang, Z. Ma, Y. Wang, J. He, A. L. Antaris, Adv. Mater. 2018, 30, 1705799.

[4] a) D. J. Naczynski, M. C. Tan, M. Zevon, B. Wall, J. Kohl, A. Kulesa, S. Chen, C. M. Roth, R. E. Riman, P. V. Moghe, Nat. Commun. 2013, 4, 2199; b) A. L. Antaris, J. T. Robinson, O. K. Yaghi, G. Hong, S. Diao, R. Luong, H. Dai, ACS Nano 2013, 7, 3644; c) D. Franke, D. K. Harris, O. Chen, O. T. Bruns, J. A. Carr, M. W. Wilson, M. G. Bawendi, Nat. Commun. 2016, 7, 12749.

[5] Kenry, Y. Duan, B. Liu, Adv. Mater. 2018, 30, 1802394.

[6] J. Luo, Z. Xie, J. W. Y. Lam, L. Cheng, H. Chen, C. Qiu, H. S. Kwok, X. Zhan, Y. Liu, D. Zhu, B. Z. Tang, Chem. Commun. 2001, 1740-1741.

[7] a) J. Mei, N. L. C. Leung, R. T. K. Kwok, J. W. Y. Lam, B. Z. Tang, Chem. Rev. 2015, 115, 11718; b) D. Wang, B. Z. Tang, Acc. Chem. Res. 2019, 52, 2559-2570; c) D. Wang, M. M. S. Lee, W. Xu, R. T. K. Kwok, J. W. Y. Lam, B. Z. Tang, Theranostics 2018, 8, 4925; d) J. Li, J. Wang, H. Li, N. Song, D. Wang, B. Z. Tang, Chem. Soc. Rev. 2020, 49, 1144-1172.

[8] a) Z. Sheng, B. Guo, D. Hu, S. Xu, W. Wu, W. H. Liew, K. Yao, J. Jiang, C. Liu, H. Zheng, B. Liu, Adv. Mater. 2018, 30, 1800766; b) Z. Zhang, X. Fang, Z. Liu, H. Liu, D. Chen, S. He, J. Zheng, B. Yang, W. Qin, X. Zhang, C. Wu, Angew. Chem. Int. Ed. 2020, 59, 3691-3698; c) J. Du, S. Liu, P. Zhang, H. Liu, Y. Li, W. 
He, C. Li, J. H. C. Chau, R. T. K. Kwok, J. W. Y. Lam, L. Cai, Y. Huang, W. Zhang, J. Hou, B. Z. Tang, ACS Appl. Mater. Inter. 2020, 12, 8040-8049; d) S. Liu, C. Chen, Y. Li, H. Zhang, J. Liu, R. Wang, S. T. H. Wong, J. W. Y. Lam, D. Ding, B. Z. Tang, Adv. Funct. Mater. $2020,30,1908125$.

[9] a) D. Wang, M. M. S. Lee, W. Xu, G. Shan, X. Zheng, R. T. K. Kwok, J. W. Y. Lam, X. Hu, B. Z. Tang, Angew. Chem. Int. Ed. 2019, 131, 5684-5688; b) M. Kang, C. Zhou, S. Wu, B. Yu, Z. Zhang, N. Song, M. M. S. Lee, W. Xu, F. J. Xu, D. Wang, L. Wang, B. Z. Tang, J. Am. Chem. Soc. 2019, 141, 16781-16789; c) H. Chen, S. Li, M. Wu, Kenry, Z. Huang, C. S. Lee, B. Liu, Angew. Chem. Int. Ed. 2020, 59, 632-636; d) C. Chen, X. Ni, S. Jia, Y. Liang, X. Wu, D. Kong, D. Ding, Adv. Mater. 2019, 31, 1904914.

[10] a) R. Zhang, Y. Duan, B. Liu, Nanoscale 2019, 11, 19241-19250; b) G. Feng, B. Liu, Acc. Chem. Res. 2018, 51, 1404-1414; c) B. Gu, K. T. Yong, B. Liu, Small Methods 2018, 2, 1700392

[11] H. S. Jung, J. H. Lee, K. Kim, S. Koo, P.Verwilst, J. L. Sessler, C. Kang, J. S. Kim, J. Am. Chem. Soc. 2017, $139,9972-9978$.

[12] Q. Dou, C. P. Teng, E. Ye, X. J. Loh, Int. J. Nanomed. 2015, 10, 419.

[13] S. Liu, X. Zhou, H. Zhang, H. Ou, J. W. Y. Lam, Y. Liu, L. Shi, D. Ding, B. Z. Tang, J. Am. Chem. Soc. 2019, 141, $5359-5368$.

[14] a) F. Paris, Z. Fuks, A. Kang, P. Capodieci, G. Juan, D. Ehleiter, A. Haimovitz-Friedman, C. Cordon-Cardo, R. Kolesnick, Science 2001, 293, 293-297; b) Y. D. Jung, M. S. Kim, B. A. Shin, K. O. Chay, B. W. Ahn, W. Liu, C. D. Bucana, G. E. Gallick, L. M. Ellis, Br. J. Cancer 2001, 84, 844-850. 


\section{Entry for the Table of Contents}

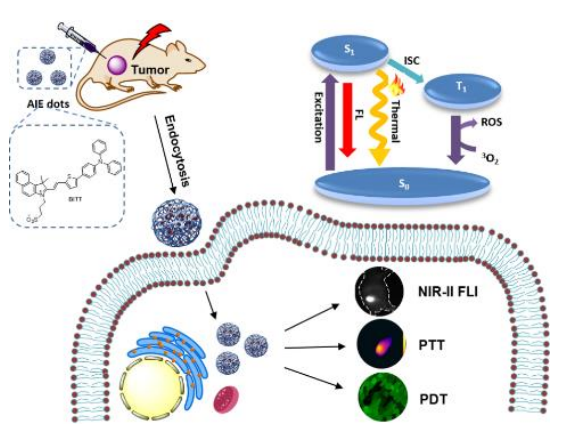

A series of NIR-emissive zwitterionic AIEgens were rationally designed and facilely constructed for the first time. One of these AlEgens exhibits long emission wavelength, effective ROS generation, and high photothermal conversion efficiency, endowing it with excellent performance in NIR-II FLI-guided synergistic PDT-PTT phototherapies. 